\title{
Clinical Implication of Ivabradine Administration Before Non-Cardiac Surgery
}

\author{
Teruhiko Imamura
}

\section{To the Editor}

Clinical use of ivabradine, which purely decreases heart rate without affecting hemodynamics, is receiving great concern. Banerjee and colleagues demonstrated a significant attenuation of heart rate response to stressful interventions including laryngoscopy, intubation, and surgical incision by using ivabradine [1]. Several concerns have been raised.

The initial concern is an optimal patient selection. Ivabradine seems to have clinical implication in improving mortality and morbidity particularly for those with chronic heart failure with reduced ejection fraction or coronary heart disease, by saving cardiac potential energy, optimizing left ventricular filling, and facilitating cardiac reverse remodeling [2]. Could the authors display comorbidity data and perform a sub-analysis among those with each comorbidity? For example, the hemodynamic responses and outcomes following the surgical interventions would be different between those with and without concomitant heart failure.

The second concern is a clinical outcome. The authors observed a hemodynamic responses following the surgical interventions between those with and without ivabradine [1], whereas clinicians' concern would rather be clinical outcomes. Transient hemodynamic fluctuation might not have considerable impact among the control group cohort. Did the authors compare clinical outcomes including bleeding amounts and surgical complications, for example?

\section{Acknowledgments}

None to declare.

\section{Financial Disclosure}

None to declare.

\section{Conflict of Interest}

None to declare.

\section{Informed Consent}

Not applicable.

\section{Data Availability}

The author declares that data supporting the findings of this study are available within the article.

\section{References}

1. Banerjee A, Mishra S. Use of preoperative single dose ivabradine for perioperative hemodynamic stabilization during non-cardiac elective surgery under general anaesthesia: a pilot study. J Clin Med Res. 2021;13(6):343354.

2. Imamura T, Kinugawa K. Optimal heart rate modulation using ivabradine. Int Heart J. 2021;62(4):717-721.

Manuscript submitted July 29, 2021, accepted August 6, 2021

Published online August 30, 2021 